\title{
Event attributes and the content of psychotic experiences in first-episode psychosis
}

\author{
DAVID RAUNE ${ }^{1}$, PAUL BEBBINGTON ${ }^{2 *}$, GRAHAM DUNN ${ }^{3}$ \\ AND ELIZABETH KUIPERS ${ }^{4}$ \\ ${ }^{1}$ Department of Adult Clinical Psychology, Acute Directorate, Ealing Locality, John Conelly Wing, West \\ London Mental Health Trust, London, UK $;^{2}$ Royal Free \& University College London Medical School, \\ UCL - Department of Mental Health Sciences (Bloomsbury Campus), Wolfson Building, London, UK; \\ ${ }^{3}$ Division of Epidemiology and Health Sciences, University of Manchester, Stopford Building, Manchester, \\ UK; ${ }^{4}$ Department of Psychology, Institute of Psychiatry, King's College London, De Crespigny Park, \\ London, UK
}

\begin{abstract}
Background. Previous psychosocial stress research, contemporary cognitive models, and new cognitive behavioural treatments for psychosis converge in suggesting that stressful events influence the content of psychotic experiences. In this paper we test whether the attributes of stressful events preceding the initial onset of psychosis are associated with core themes of the illness.
\end{abstract}

Method. Forty-one people who had experienced a first episode of psychosis were assessed on the attributes of stressful events occurring in the year before onset, the themes (persecutory, depressive, and grandiose) associated with their delusions and the content of their auditory hallucinations.

Results. Principal component analysis yielded four components accounting for $72 \%$ of the variance. As hypothesized, intrusive events were associated with the development of delusions with persecutory themes. Grandiose delusions were negatively associated with loss events. Depressive delusions appear to be associated with danger events rather than loss events.

Conclusion. There are links between stressful event attributes and core psychotic themes at firstepisode psychosis. This has implications for theoretical models of, and early psychological intervention for, psychosis.

\section{INTRODUCTION}

The contents of psychotic experience were typically regarded as incomprehensible for much of the last century (Jaspers, 1913). Berrios (1996) famously described delusions as being 'empty speech acts'. However, psychotic experience is certainly characterized by common themes. Spitzer (1992) pointed out that the range of themes apparent in psychosis around the world is narrow, the most common being persecutory, depressive and grandiose. Themes represent the

\footnotetext{
* Address for correspondence: Professor Paul Bebbington, Royal Free \& University College London Medical School, UCL Department of Mental Health Sciences (Bloomsbury Campus), Wolfson Building, 48 Riding House Street, London W1W 7EY, UK. (Email: p.bebbington@ucl.ac.uk)
}

particular cognitive affective meaning or schema that individuals use to organize their relationship to self and the world (Lebowitz \& Newman, 1996). They are important theoretically for models of psychosis, and clinically because they are linked to a range of other psychopathological phenomena, even during the first episode (e.g. Fowler, 2000).

Since the mid-1960s, studies of stressful events in psychosis have shown that events cluster significantly in the weeks or months before illness (Bebbington et al. 1995). This finding has traditionally been interpreted as a non-specific triggering effect. However, psychosocial stress studies (Morrison et al. 2003), contemporary cognitive models (Garety et al. 
2001), and new cognitive behavioural treatments (Fowler, 2000) have recently converged in suggesting that stressful life events might actually shape the content of psychosis. Empirical studies have hinted that events might specifically influence the themes of delusions and auditory hallucinations (Morrison et al. 2003; Hardy et al. 2005). Further, Morrison et al. (2003) have hypothesized that there may be developmental processes common to psychosis and posttraumatic stress disorder (PTSD). Thus, just as in PTSD, the content of the intrusive psychotic symptoms (delusions and auditory hallucinations) may reflect the attributes of prior stressful events. Indeed, these authors have argued that it is only the degree of self-evidence (to both patient and clinician) of links between symptom content and past stressful events that determines which diagnosis is given (i.e. PTSD or psychosis) (Morrison et al. 2003). Garety et al. (2001) proposed that certain attributes of aversive environments may encourage particular types of psychological appraisal. They also suggested that adverse events may change people's core beliefs about themselves and the world, and that this is likely to influence psychotic content.

Harris (1987) developed the concept of the intrusive or 'paranoia-inducing' event, and found that patients who had schizophrenia were 20 times more likely than controls to have experienced an intrusive event in the 3 weeks before onset or relapse. However, she did not link intrusive events directly to persecutory themes. Authors of cognitive models of persecutory themes have discussed a specific role for intrusive events (Bentall et al. 2001, pp. 1151-1152; Trower, 2003, p. 242), while Freeman et al. (2002, p. 335) postulate that stressful events encourage people to see the world as hostile or threatening. Intrusive events should, therefore, be associated particularly with persecutory themes. Given the various potential roles of anxiety in persecutory delusions (Freeman et al. 2002) it is also possible that danger events would predict persecutory themes.

Non-psychotic depression has been linked theoretically with the experience of loss (e.g. Brown \& Harris 1989a), and it is reasonable to suppose that loss events, broadly conceived (loss, self-esteem, and humiliation events), would equally predict the development of psychotic depressive themes. Paradoxically, loss has also been associated with grandiosity through the idea of manic defence (Thomas \& Bentall, 2002).

Empirical support for the idea that stressful life events directly influence the content of psychosis would have implications both for theoretical models of psychosis and for techniques of early intervention. Although suggestive, the psychosocial stress literature linking events to psychosis - on which cognitive models are partly predicated-has typically suffered from methodological inadequacies and limitations (Bebbington et al. 1995; Morrison et al. 2003). The empirical evidence is consequently weak. The influence of stressful life-event attributes on psychotic themes should moreover be tested at the first episode of psychosis rather than at relapse. This is because later in the course other variables might disrupt or camouflage any event-theme relationship (e.g. Yung, 1998). Additionally, the concept of sensitization (see Ramana \& Bebbington, 1995) would predict that any 'lock and key' relationship between stressful-event attributes and psychotic themes would be easier to detect at the first episode: the subsequent re-emergence of a theme might be triggered by non-specific psychosocial stress. Studies of later episodes bear more relevance to theme maintenance than to original cause.

We therefore chose to test hypotheses about the relationship between events and symptom content in a sample of people experiencing firstepisode psychosis. We predicted primarily that events with the qualities either of intrusiveness or of danger would be particularly associated with persecutory psychotic experiences. Second, we predicted that themes both of depression and of grandiosity would be associated with events having attributes of loss, humiliation and the lowering of self-esteem.

\section{METHOD}

All new cases of functional psychotic disorders referred to the south London catchment areas of East Lambeth, South Southwark and Croydon were assessed for inclusion in the study. Inclusion criteria included an ICD-10 diagnosis of psychosis based on a SCAN interview and the 
application of the CATEGO program. All participants were aged between 16 and 65 years, and spoke English. They had experienced the onset of their disorder within the 12-month period before interview. Onset was defined as the first emergence of delusions, hallucinations, or formal thought disorder, and had to be datable within 1 week. Patients with gross organic pathology, or moderate or severe learning disability were excluded. Forty-six people met our criteria during the period of the study, of whom five refused to participate, giving a refusal rate of $11 \%$. People who refused to participate were slightly older than those who agreed, and four of the five were male. Of the 41 participants, 40 had delusions, and 26 experienced auditory hallucinations.

\section{Procedure}

There were 26 referral units, covering the Maudsley, Bethlem, Mayday and Warlingham hospitals in south London and all their associated community mental health teams. All community teams included in the study were contacted at least once a week, and all wards three times a week. The local bed manager (responsible for arranging admissions to hospital) was contacted on a daily basis.

\section{Diagnosis and assessment of the mental state}

Information about the participants' sociodemographic characteristics and various details of their symptoms were collected in a standard format. SCAN 1.1 (WHO, 1992) was used to establish the respondents' psychopathological status. This well-known instrument includes the 10th edition of the Present State Examination, and covers an exhaustive range of neurotic and psychotic symptoms. Data elicited through this semi-structured interview were then submitted to the CATEGO-V diagnostic program. Symptoms were elicited in relation to the peak of the psychotic episode in order to obtain the most representative pattern. In addition to its diagnostic facility, SCAN was used as the basis for identifying the themes present in the person's delusions and the content of their auditory hallucinations. This instrument is particularly suitable for the purpose because of the detailed distinction it makes between different types of delusions, and between delusions and anomalous experiences.

\section{Identification of psychotic themes and contents}

In this paper, we analyse three sets of themes and contents, persecutory, depressive and grandiose. We also identified a referential theme, but this did not permit analysis as it was present in almost all participants.

A persecutory delusional theme was rated as present if a score of at least 2 on the standard 1-3 scale was given for the SCAN items delusions of persecution or delusions of conspiracy. Delusions of reference were not in themselves taken as an indication of beliefs in persecution. Persecutory voice content was operationalized as a voice speaking of the person being harmed. A depressive delusional theme was identified in the present study from a rating of $\geqslant 2$ on depressive delusions of reference, depressive delusions of guilt, delusions of catastrophe, or depressive hypochondriacal delusions. A depressive voice content was rated if the content was consistent with these delusional themes. A grandiose delusional theme was considered present if a rating of at least 2 was given for the expansive reference delusions, or delusions of grandiose ability or of grandiose identity. Grandiose voice content was rated if the content was consistent with these SCAN items.

\section{Assessment of stressful life events}

A history of life events in the 12 months before illness onset was established using the Life Events and Difficulties Schedule (LEDS 2; Brown \& Harris 1989b), a semi-structured interview with a proven record in the study of psychosis (Brown \& Birley, 1968; Bebbington et al. 1993). Events are identified and rated from their likely meaning of the events to the participants. The LEDS involves a rigorous crossexamination, and accounts of events that might have been delusional were excluded from the analysis if they could not to some extent be corroborated by accounts from formal or informal carers. Participants were interviewed after it was felt insight had returned, and carers' accounts were subjected to a similar level of scrutiny through internal and external validity checks. Very few events were rejected as being delusional. Life events were rated on a number of dimensions. 
The independence of the event is a judgement of how much it could have been influenced by any behaviour of the person conditional on the fact of the disorder under investigation. Events were analysed if they fell into the categories 'independent' or 'possibly independent'.

Threat is rated by the researcher according to the all-round contextual unpleasantness of the event. The rating is assigned according to how an average person would find the event, given the respondent's biographical circumstances. It is rated on a 4-point scale, from 'marked' to 'little or no threat'. In this study, the long-term threat (i.e. the notional threat level 14 days after the event) was used for analysis.

A number of more specific scales have been constructed since the initial threat scale was devised. Those used in the present study were Loss, Danger, Humiliation, Intrusiveness, and a new scale, Self-esteem. The last was developed as an extension of the Humiliation scale for the purposes of the present project. Events were rated on threat, and then, if threat was at least moderate, on loss, danger and humiliation. All events were rated on intrusiveness and selfesteem, irrespective of the threat level. In one third $(14 / 41)$ of cases, the reliability of the LEDS scores was checked informally by Tirril Harris and a panel of raters, who deemed the ratings acceptable.

Loss was only rated if the event was rated at least 2 'Moderate threat'. Scale guidelines identify four types of external loss: loss of material possessions/money; loss of person; loss of community respect; or loss of employment. Internal loss relates to loss of a 'cherished idea' about the self or others. Loss is rated on a 4-point scale.

Danger events are those that suggest the possibility of a future loss. Danger events can be classified along the lines of the loss categories. Two factors are taken into account when rating it: the unpleasantness of the event and the likelihood of it actually happening. Danger is only rated if the event is rated as having at least moderate long-term contextual threat. It is rated on a 4-point scale.

Humiliating events involve the person being socially devalued in relation to self or others. Usually only events involving core ties are included, that is, people very close to the individual. However, if an event is particularly shameful, other people can be included. Only events rated as having at least moderate longterm contextual threat are included. Unlike the other scales, humiliation is rated only as present/absent.

Intrusive events involve interference and attempted control of the subject by outsiders or people where there is no evidence of closeness. Usually but not always, intrusive events involve harmful consequences, and will often be committed by a figure of authority. It is rated on a 4-point scale, irrespective of the threat rating.

Self-esteem is an evaluative component of the self-concept. The Self-esteem event scale was developed by D.R. from the work of Bentall et al. (1994). Self-esteem reducing events are those that either deprive the self of an important source of worth or involve a situation that actively devalues self-worth. Such events would be expected to reduce the overall value individuals place on themselves. There are two broad classes of such events. These are, first, events caused by the respondents themselves. These will often involve personal failure in areas such as education, work, reproduction or relationships. The importance of the failure, and how directly personal shortcomings are implicated, will influence the rating. A second class of events that lower self-esteem are those caused by other people. Such events will often also meet the criteria of Brown et al. (1995) for humiliation. However, whilst what is humiliating will always reduce self-esteem (under the Brown et al. 1995 criteria), what lowers self-esteem may not always be humiliating. Humiliation is just one way of lowering selfesteem. It is also true that a given event that is both humiliating and self-esteem reducing will not necessarily receive the same severity rating for each quality. Because the self-esteem rating is new, we tested inter-rater reliability with Tirril Harris. There was agreement on the 4-point scale in 19 out of 20 events, and perfect agreement about which events were rated moderate or severe.

\section{Analysis}

This dataset poses problems in analysis because there is considerable overlap between psychotic themes and contents. Moreover, individual participants may experience numerous events, each event may be rated on more than one 
Table 1. Characteristics of sample

\begin{tabular}{lccc}
\hline \hline & $\begin{array}{c}\text { Male } \\
n=24 \\
(58 \cdot 5 \%)\end{array}$ & $\begin{array}{c}\text { Female } \\
n=17 \\
(41 \cdot 5 \%)\end{array}$ & $\begin{array}{c}\text { Overall } \\
n=41 \\
(100 \%)\end{array}$ \\
\hline $\begin{array}{l}\text { Age at onset (years) } \\
\text { Mean (s.D.) }\end{array}$ & $29 \cdot 5(11 \cdot 8)$ & $29 \cdot 8(7 \cdot 8)$ & $29 \cdot 6(11 \cdot 1)$ \\
$\begin{array}{l}\text { Illness length (weeks) } \\
\text { Mean (s.D.) }\end{array}$ & $20 \cdot 0(14 \cdot 3)$ & $14 \cdot 7(6 \cdot 4)$ & $17 \cdot 2(11 \cdot 8)$ \\
$\quad$ Median & $17 \cdot 5$ & $14 \cdot 1$ & $15 \cdot 4$ \\
$\begin{array}{l}\text { Diagnosis } \\
\text { Schizophrenia/ } \\
\text { schizoaffective }\end{array}$ & $14(58 \cdot 3 \%)$ & $11(64 \cdot 7 \%)$ & $25(61 \cdot 0 \%)$ \\
$\quad$ Bipolar & $6(25 \cdot 0 \%)$ & $2(11 \cdot 8 \%)$ & $8(19 \cdot 5 \%)$ \\
$\quad$ Other psychoses & $4(16 \cdot 7 \%)$ & $4(23 \cdot 5 \%)$ & $8(19 \cdot 5 \%)$ \\
Ethnicity & & & \\
$\quad$ Black & $10(41 \cdot 7 \%)$ & $8(47 \cdot 1 \%)$ & $18(43 \cdot 9 \%)$ \\
$\quad$ White & $13(54 \cdot 2 \%)$ & $9(52 \cdot 9 \%)$ & $22(53 \cdot 7 \%)$ \\
$\quad$ Unclassified & $1(4 \cdot 1 \%)$ & 0 & $1(2 \cdot 4 \%)$ \\
\hline \hline
\end{tabular}

attribute, and different events will have different ratings. As a way of reducing this information, we condensed events into characteristics of the participants, i.e. 'this person has had event(s) with the following characteristics', using the highest rating on each event attribute of any event experienced. We then relied primarily on principal components analysis, followed by varimax rotation, as a technique for eliciting patterns in line with our hypotheses. The technique allows both event presence and event severity to influence the factor loadings. Delusions and auditory hallucinations were analysed separately because of the possibility that the relationship between events and themes might differ in relation to different symptoms.

\section{RESULTS}

The characteristics of the sample are shown in Table 1 . The mean age at onset was in line with other studies, although it did not differ as expected by sex. The median length of illness was very short because the onset had to be datable and to have occurred within a year of the research assessment. The range was from 9 days to 52 weeks. Most diagnoses were of schizophrenia, with appreciable minorities of bipolar and schizoaffective disorder.

The distribution of themes in delusions and auditory hallucinations is shown in Table 2 . The degree of overlap between themes is clear, although the combination of grandiose and
Table 2. Prevalence of themes in delusions $\left(n=39^{\mathrm{a}}\right)$ and auditory hallucinations $(n=26)$

\begin{tabular}{lcc}
\hline \hline & Delusion & $\begin{array}{c}\text { Auditory } \\
n(\%)\end{array}$ \\
Theme & $34(87)$ & $n(\%)$ \\
\hline Persecutory & $17(43)$ & $16(62)$ \\
Grandiose & $11(28)$ & $2(8)$ \\
Depressive & $13(33)$ & $11(42)$ \\
Persecutory and grandiose & $11(28)$ & 0 \\
Persecutory and depressive & $3(8)$ & $11(42)$ \\
Grandiose and depressive & 0 \\
\hline \hline
\end{tabular}

a One person's delusional theme was unclassifiable.

Table 3. Prevalence of stressful event attributes $(n=41)$

\begin{tabular}{|c|c|c|c|}
\hline Attribute & $n(\%)$ & Combination of attributes & $n(\%)$ \\
\hline Humiliation & $19(46)$ & $\begin{array}{l}\text { Intrusive event plus humiliation } \\
\text { event }\end{array}$ & $16(39)$ \\
\hline Loss & $26(63)$ & Intrusive event plus loss event & $21(51)$ \\
\hline Danger & $31(76)$ & Intrusive event plus danger event & $25(61)$ \\
\hline Self-esteem & $29(71)$ & $\begin{array}{l}\text { Intrusive event plus self-esteem } \\
\text { event }\end{array}$ & $26(63)$ \\
\hline Intrusiveness & $30(73)$ & Intrusive event alone & $2(6)$ \\
\hline
\end{tabular}

depressive themes was rare, as might be expected. A similar overlap in stressful-event attributes is apparent in Table 3. Events with our chosen characteristics were common, probably partly because the sample was defined by the existence of a psychotic disorder. Indeed, three quarters of those who developed persecutory delusions or hallucinations had experienced intrusive events in the period before onset. Participants commonly had events with more than one sort of attribute.

Because of this overlap of both themes and event attributes, we considered that the most straightforward way of making sense of our data was to use principal components analysis. We entered into the analysis the content of auditory hallucinations, the themes of delusions, and the attributes of events. In Table 4, we present the correlation matrix for the phenomenological variables and the life- event attributes, which again emphasizes the issue of overlap. (Note that where variables are dichotomous, Pearson's $r$ is the equivalent of a $\varphi$ coefficient). This matrix reveals a sufficient pattern of magnitudes and significances to indicate that there is indeed some underlying 


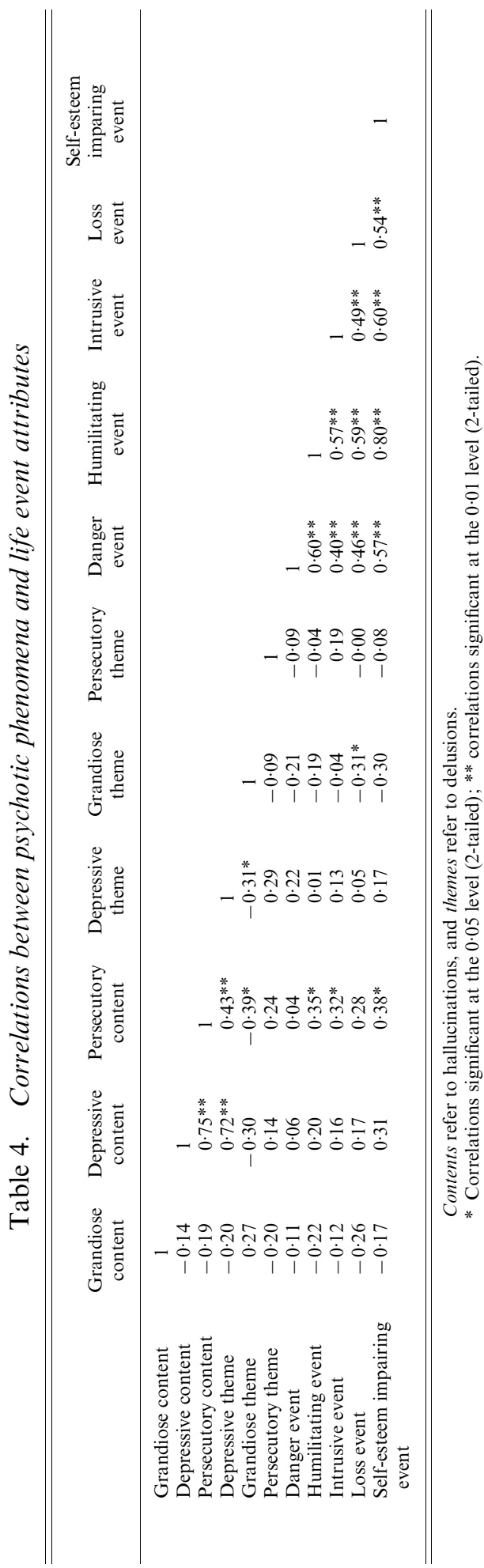

structure, sufficient to support at least tentative conclusions from the principal components analysis.

The principle components analysis yielded a model comprising four components with unrotated eigenvalues $>1$, capable of explaining $72 \%$ of the variance (Table 5). After varimax rotation, these components can be interpreted in clinical terms. Component $1(30.7 \%$ of the variance post-rotation) is a reflection of the close relationship between event attributes. It loads strongly on all life-event attributes, particularly those associated with humiliation and lowered self-esteem, and weakly on auditory hallucinations with persecutory or depressive content. It also loads negatively but weakly on grandiose delusions. Component 2 ( $18.6 \%$ of the variance) loads strongly on auditory hallucinations with depressive and persecutory content, and on persecutory delusions, much less so on depressive delusions, and negatively on grandiose delusions. It loads positively on intrusive events and negatively on danger events. Component $3(11.7 \%$ of the variance $)$ loads strongly on grandiose delusions, and weakly negatively on depressive auditory hallucination content and loss events. It has a weak positive loading on persecutory delusions, but a weak negative loading on persecutory hallucinations. Component 4 (11.2\% of the variance) relates strongly to depressive delusions, weakly to persecutory delusions, and moderately to danger events. There is little loading on auditory hallucinations in this component.

\section{DISCUSSION}

Studies of stressful events in psychosis have rarely assessed event attributes of a higher conceptual order (e.g. humiliation), focusing rather on lower order typologies (e.g. their relationship to issues concerning housing or finance). The seminal study of Harris (1987) was based on a post-hoc re-analysis of existing data, which weakens the validity of her results. Many studies have used inadequate methods to assess life events (e.g. see Morrison et al. 2003). They have also restricted themselves to the final 3 weeks or 3 months before onset (e.g. Harris, 1987). While this is suitable for testing a triggering hypothesis, the capacity for events to shape cognitive themes may operate over a longer time-frame. 
Table 5. Principal components analysis of psychotic themes and life-event attributes

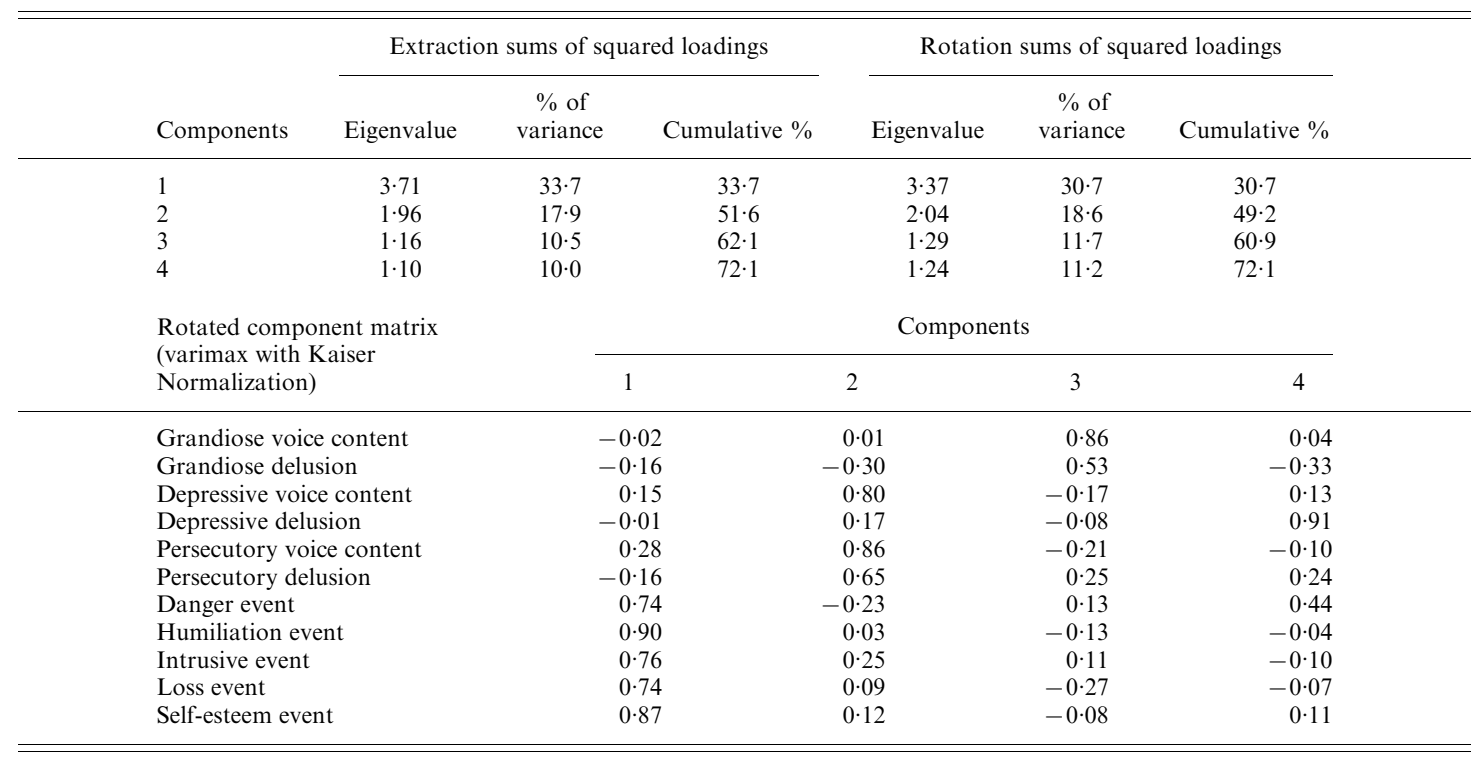

The problem that a given event often carries more than one attribute (e.g. intrusiveness and loss) has not been addressed in the life-event/psychosis literature. Neither has the concurrence of the different psychosis themes (e.g. persecutory and grandiose). It is, therefore, difficult to disentangle which stressful-event attribute might have influenced which psychotic theme. There are other ways of analysing this complex dataset. One would be to concentrate only on the attributes of the event closest in time to onset. We did not choose to do this because it would neglect the potential impact of events with particular attributes even when they were followed by events with other attributes. Another would be to include earlier events only if they occurred within a set period ( 3 months for example) of the latest event, but this would be a procedure both complex and arbitrary.

Our study is of an exclusively first-episode sample, and consequently has a short length of illness (median 17 weeks). In this respect, the sample is similar to that of Drake et al. (2000). Because we were interested in process rather than diagnosis, we included people with the whole range of psychotic diagnoses. Our approach was in the psychosocial rather than the (complementary) cognitive tradition. In other words we were not primarily concerned with our subjects' own interpretation of events, but with an attempt to objectify their characteristics using operational definitions. We restricted ourselves to the analysis of events occurring in the year before onset. Thus, we focused on the stress component of a putative stressvulnerability model. We acknowledge that vulnerability may have many components, including earlier events that can be equally formative (Bebbington et al. 2004). We tested a priori hypotheses using the LEDS, a wellestablished event-assessment instrument. We assessed core psychopathological themes, and applied principal components analysis in a novel way to tackle the overlap of themes and event attributes. We used varimax rotation for its relative ease of interpretation. Although this form of rotation can distort conclusions by forcing orthogonality, in practice the components in our analysis were not greatly correlated (ranging from 0 to $0 \cdot 16$ ).

Because our numbers were relatively limited for an analysis of this type, we constructed lifeevent variables that reflected merely whether subjects had experienced an event of a given type. This meant that we took no account of the fact that many people had experienced multiple events of the same type. It is thus possible that our results underestimated the strength of the relationship between stressful-event attributes and psychotic themes. However, it can also be 
argued that this is a legitimate way of formulating the participants' experience, at least as valid as a cumulative measure of events of a particular attribute. Attempts to quantify the cumulative impact of stressful events preceding episodes of depression have not been very successful. It is almost guaranteed that the process of defining psychotic themes and event attributes actually simplifies the texture of experience in a way that disguises the meaningful connections between its external and internal aspects. If so, even weak links between artificially narrowed constructs may provide evidence of meaningful connections. Other limitations of the study include its cross-sectional design and the fact that the data were collected by a single researcher. Thus, our results are merely indicative, and most certainly require replication. Moreover, the psychosocial approach adopted in this study should be complemented by cognitive studies examining peoples' subjective interpretations of events.

The largest component in the present analysis (31\% of variance) predominantly links the attributes of the life events, and reflects the strong overlap between the attributes rated and perhaps a degree of commonality deriving from the fact of being established through the same instrument. It also links events of all types, albeit weakly, with depressive and persecutory hallucinations. This has parallels with recent findings suggesting traumatic events seem to have a particularly strong relationship with hallucinations (Hardy et al. 2005). The remaining three components also provide interpretable links between event attributes and psychopathological themes. The components may also indicate more general patterns of first-episode psychosis psychopathology. Thus persecutory delusions are linked both to depressive and to grandiose delusions, but there is little linkage between the two last types (Tables 2 and 4). Likewise there is some separation between delusions and auditory hallucinations, perhaps corresponding to the distinction between delusional states and nuclear schizophrenia. There are instances where this results in differential relationships with life-event characteristics.

\section{Persecutory themes}

Our results offer support for the hypothesis linking intrusiveness and persecutory themes.
Thus, the second component (accounting for around $18 \%$ of the variance) primarily incorporated persecutory delusions, persecutory and depressive auditory hallucinations, and intrusive events. There was no loading of intrusive events with Component 3, which loaded heavily on grandiosity, nor was there much of a loading on Component 4, which largely reflected depressive delusional states. There may, therefore, be some specificity in the relationship between intrusive events and persecutory delusions. However, intrusive events were not specifically associated with persecutory voices, as depressive voice content also formed part of Component 2 (cf. Read et al. 2003, p. 12). Many of the intrusive events were at least moderately severe, and included physical assault, being threatened with a knife, a child relative being given crack cocaine, attending crown court after an attempted rape, sexual disease infection, being burgled, and police arrest of a close relative. Thus, for persecutory delusions, these sorts of intrusive stressful events may indeed be capable of skewing people's fundamental schemas about the world (Garety et al. 2001). Intrusive events might induce an external, specific, personal attributional style (Kinderman \& Bentall, 1997), suspicions about other people's intentions (Frith, 2004), a self-perception of vulnerability (Freeman et al. 2002), and thoughts of being compelled by powerful others (Birchwood et al. 2000). Our results support Birchwood's (1999) warning about the dangers of cognitive modelling of delusions in isolation from the social context in which they occur.

The support for our prediction that danger events would influence persecutory themes was ambiguous. The main component on which persecutory delusions and voices loaded (Component 2) actually had a negative relationship with danger events. Component 4 linked depressive and persecutory delusions with danger events, although depressive delusions had by far the highest loading. This may mean that intrusive events have a more specific and direct influence on persecutory themes than anxiety-inducing events of a less specific nature. This might be due to the specific implications of intrusive events for a person's interpersonal schemata, at least in terms of the original causation (if not the later maintenance) of persecutory themes. 


\section{Depressive themes}

We found no indication that loss events, broadly conceived (loss, self-esteem, and humiliation events) are associated with the development of depressive psychotic themes. In contrast, we found that danger events were clearly linked to depressive delusions (Component 4). This relationship is specific to danger events, since depressive delusions were not associated with any other type of event. Given that danger events by definition have connotations that may persist for weeks or months, it is possible that in some cases they lead to a psychosis with a depressive delusional theme. In contrast, danger events were in fact negatively related to depressive auditory hallucinations, suggesting a clear difference between these symptom types. In Component 2, intrusive events were associated with depressive voices.

\section{Grandiose themes}

Grandiosity has been explained in terms of a manic defence (e.g. Thomas \& Bentall, 2002), but the present study did not find that loss, humiliation or self-esteem events were related to grandiose themes. The result is clear: Component 3 loaded strongly on grandiose delusions, and negatively on events characterized by loss. Two recent studies have reported other evidence against the defence hypothesis, finding that grandiosity is related to high self-esteem, and is inversely related to negative schemata about the self (Smith et al. 2005; B. Smith et al. unpublished observations). It thus appears that grandiose delusions may be a way of improving and maintaining selfesteem, regardless of whether it was initially low.

\section{CONCLUSION}

Our study thus found modest relationships between prior stressful event attributes and themes in first-episode psychosis. Persecutory delusions and voices were linked to intrusive events, depressive delusions were linked to danger events, depressive auditory hallucinations were related to intrusive events (perhaps because of overlap with persecutory voice content), and grandiose delusions were associated primarily with an absence of loss events. The relationship between persecutory themes and intrusive events corroborated an a priori hypothesis, but the strongest link statistically was between depressive delusions and danger events, which we did not predict. It appears that different psychotic symptoms (auditory hallucination, cf. delusions) may differ in their strength of relationship with stressfulevent attributes, and intriguingly this may depend on which psychotic theme is examined.

Nevertheless, if life-event characteristics are related to the content of subsequent delusions and auditory hallucinations, this goes some way to refute the idea that symptoms of psychosis are without meaning, and certainly has clinical implications. Incorporating recent stressful events into the assessment and psychological formulation of core psychotic themes may assist with patient engagement, normalization, and insight. Helping an individual to 'make sense' of how previous events relate to current symptoms should reduce anxiety, depression and feelings of powerlessness, all of which seem to be associated with psychological appraisals (C. Green et al. unpublished observations). This ability to reappraise symptoms as an unusual but understandable reaction to normal events is an important component of CBT for psychosis (e.g. Fowler et al. 1995; Fowler, 2000). The evidence from this study suggests that such processes have a theoretical as well as a clinical basis. It also provides a rationale for early intervention for prodromal symptoms of psychosis, and informs the strategies that might be used (e.g. Morrison et al. 2004).

\section{ACKNOWLEDGEMENTS}

Thanks are due to the participants and to the 26 ward and community mental health teams of the South London and Maudsley National Health Service Trust from which patients were recruited. We also thank Tirril Harris and her team for assistance with life event ratings. D.R. was supported by a Medical Research Council studentship.

\section{DECLARATION OF INTEREST}

None. 


\section{REFERENCES}

Bebbington, P. E., Bhugra, D., Brugha, T., Farrell, M., Lewis, G., Meltzer, H., Jenkins, R., Lewis, G. \& Meltzer, H. (2004). Psychosis, victimisation and childhood disadvantage: evidence from the Second British National Survey of Psychiatric Epidemiology. British Journal of Psychiatry 185, 220-226.

Bebbington, P. E., Bowen, J., Hirsch, S. R. \& Kuipers, E. A. (1995). Schizophrenia and psychosocial stress. In Schizophrenia (ed. S. R. Hirsch and D. R. Weinberger), pp. 587-604. Blackwell: Oxford, England.

Bebbington, P. E., Wilkins, S., Jones, P., Foerster, A., Murray, R. M., Toone, B. \& Lewis, S. (1993). Life events and psychosis: initial results from the Camberwell Collaborative Psychosis Study. British Journal of Psychiatry 162, 72-79.

Bentall, R. P., Corcoran, R., Howard, R., Blackwood, N. \& Kinderman, P. (2001). Persecutory delusions: a review and theoretical integration. Clinical Psychology Review 21, 1143-1192.

Bentall, R. P., Kinderman, P. \& Kaney, S. (1994). The self, attributional processes and abnormal processes and abnormal beliefs: towards a model of persecutory delusions. Behaviour Therapy and Research 32, 331-341.

Berrios, H. (1996). The History of Mental Symptoms: Descriptive Psychopathology Since the Nineteenth Century. Cambridge University Press: Cambridge.

Birchwood, M. (1999). Commentary on Garety \& Freeman I: 'Cognitive approaches to delusions - a critical review of theories and evidence'. British Journal of Clinical Psychology 38, 315-318.

Birchwood, M., Meaden, A., Trower, P., Gilbert, P. \& Plaistow, J. (2000). The power and omnipotence of voices: subordination and entrapment by voices and significant others. Psychological Medicine 30, 337-344.

Brown, G. W. \& Birley, J. L. T. (1968). Crises and life changes and the onset of schizophrenia. Journal of Health and Social Behaviour 9, 203-214.

Brown, G. W. \& Harris, T. O. (1989a). Life Events and Illness. Unwin Hyman: London.

Brown, G. W. \& Harris, T. O. (1989b). The Life Events and Difficulties Schedule 2 (LEDS2). Royal Holloway and Bedford New College, University of London: London.

Brown, G. W., Harris, T. O. \& Hepworth, C. (1995). Loss, humiliation and entrapment in women developing depression: a patient and non-patient comparison. Psychological Medicine 25, 7-21.

Drake, R. J., Haley, C. J., Akhtar, S. \& Lewis, S. W. (2000). Causes and consequences of duration of untreated psychosis in schizophrenia. British Journal of Psychiatry 177, 511-515.

Fowler, D. (2000). Psychological formulation of early episodes of psychosis: a cognitive model. In Early Intervention in Psychosis, pp. 101-127. John Wiley \& Sons Ltd: Chichester.

Fowler, D., Garety, P. A. \& Kuipers, E. K. (1995). Cognitive Behaviour Therapy for Psychosis: Theory and Practice. John Wiley: Chichester.

Freeman, D., Garety, P. A., Kuipers, E., Fowler, D. \& Bebbington, P. E. (2002). A cognitive model of persecutory delusions. British Journal of Clinical Psychology 41, 331-347.
Frith, C. (2004). Schizophrenia and theory of mind. Psychological Medicine 34, 385-389.

Garety, P. A., Kuipers, E., Fowler, D., Freeman, D. \& Bebbington, P. E. (2001). A cognitive model of the positive symptoms of psychosis. Psychological Medicine 31, 189-195.

Hardy, A., Fowler, D., Freeman, D., Smith, B., Steel, C., Evans, J., Garety, P., Kuipers, E., Bebbington, P. E. \& Dunn, G. (2005). Trauma and hallucinatory experience in psychosis. Journal of Nervous and Mental Disease 193, 501-507.

Harris, T. (1987). Recent developments in the study of life events in relation to psychiatric and physical disorders. In Psychiatric Epidemiology (ed. B. Cooper), pp. 81-103. Croom Helm: London.

Jaspers, K. (1913). Kausale und verstellende Zusammen Länge zwischen Schicksal und Psychose bei der Dementia praecox (Schizophrenie). Zeitschrift für Neurologie 14, 158-263. (Translated J. Hoenig, in Themes and Variations in European Psychiatry (ed. S. R. Hirsch and M. Shepherd), 1994. Wright: Bristol).

Kinderman, P. \& Bentall, R. P. (1997). Causal attributions in paranoia and depression: internal, personal and situational attributions for negative events. Journal of Abnormal Psychology 106, 341-345.

Lebowitz, L. \& Newman, L. (1996). The role of cognitive-affective themes in the assessment and treatment of trauma reactions. Clinical Psychology and Psychotherapy 3, 196-207.

Morrison, A., French, P., Walford, L., Lewis, S. W., Kilcommons, A., Green, J., Parker, S. \& Bentall, R. (2004). Cognitive therapy for the prevention of psychosis in people at ultra-high risk. British Journal of Psychiatry 185, 291-297.

Morrison, A. P., Frame, L. \& Larkin, W. (2003). Relationships between trauma and psychosis: a review and integration. British Journal of Clinical Psychology 42, 331-353.

Ramana, R. \& Bebbington, P. (1995). Social influences on bioplar affective disorders. Social Psychiatry and Psychiatric Epidemiology 30, $152-160$.

Read, J., Agar, K., Argyle, N. \& Aderhold, V. (2003). Sexual and physical abuse during childhood and adulthood as predictors of hallucinations, delusions and thought disorder. Psychology and Psychotherapy: Theory, Research and Practise 76, $1-22$.

Smith, N., Freeman, D. \& Kuipers, E. (2005). Grandiose delusions: an experimental investigation of the delusion-as-defence hypothesis. Journal of Nervous and Mental Disease 193, 480- 487.

Spitzer, M. (1992). The phenomenology of delusions. Psychiatric Annals 22, 252-259.

Thomas, J. \& Bentall, R. P. (2002). Hypomanic traits and response styles to depression. British Journal of Clinical Psychology 41, 309-313.

Trower, P. (2003). Theoretical developments in REBT as applied to schizophrenia. In Rational Emotive Behaviour Therapy: Theoretical Developments (ed. W. Dryden), pp. 228-246. Bruner Routledge: Hove.

WHO (1992). SCAN: Schedules for Clinical Assessment in Neuropsychiatry. Geneva: World Health Organization.

Yung, A. R. (1998). First episode research. Current Opinion in Psychiatry 11, 27-32. 